\title{
Constraining nuclear data via cosmological observations: Neutrino energy transport and big bang nucleosynthesis
}

\author{
Mark Paris ${ }^{1, a}$, George Fuller ${ }^{2}$, Evan Grohs $^{3}$, Chad Kishimoto ${ }^{4}$, and Alexey Vlasenko ${ }^{5}$ \\ 1 Theoretical Division, Los Alamos National Laboratory, Los Alamos, New Mexico 87545, USA \\ 2 Department of Physics, University of California, San Diego, La Jolla, California 92093, USA \\ 3 Department of Physics, University of Michigan, Ann Arbor, Michigan 48109, USA \\ ${ }^{4}$ Department of Physics and Biophysics, University of San Diego, San Diego, California 92110, USA \\ 5 Department of Physics, North Carolina State University, Raleigh, North Carolina 27695, USA
}

\begin{abstract}
We introduce a new computational capability that moves toward a self-consistent calculation of neutrino transport and nuclear reactions for big bang nucleosynthesis (BBN). Such a self-consistent approach is needed to be able to extract detailed information about nuclear reactions and physics beyond the standard model from precision cosmological observations of primordial nuclides and the cosmic microwave background radiation. We calculate the evolution of the early universe through the epochs of weak decoupling, weak freeze-out and big bang nucleosynthesis (BBN) by simultaneously coupling a full strong, electromagnetic, and weak nuclear reaction network with a multi-energy group Boltzmann neutrino energy transport scheme. The modular structure of our approach allows the dissection of the relative contributions of each process responsible for evolving the dynamics of the early universe. Such an approach allows a detailed account of the evolution of the active neutrino energy distribution functions alongside and selfconsistently with the nuclear reactions and entropy/heat generation and flow between the neutrino and photon/electron/positron/baryon plasma components. Our calculations reveal nonlinear feedback in the time evolution of neutrino distribution functions and plasma thermodynamic conditions. We discuss the time development of neutrino spectral distortions and concomitant entropy production and extraction from the plasma. These effects result in changes in the computed values of the BBN deuterium and helium-4 yields that are on the order of a half-percent relative to a baseline standard BBN calculation with no neutrino transport. This is an order of magnitude larger effect than in previous estimates. For particular implementations of quantum corrections in plasma thermodynamics, our calculations show a $0.4 \%$ increase in deuterium and a $0.6 \%$ decrease in ${ }^{4} \mathrm{He}$ over our baseline. The magnitude of these changes are on the order of uncertainties in the nuclear physics for the case of deuterium and are potentially significant for the error budget of helium in upcoming cosmological observations.
\end{abstract}

\section{Introduction}

Precise forthcoming observations of observables related to the cosmic microwave background (CMB) radiation [1] and big bang nucleosynthesis [2,3] (BBN) will further constrain physics beyond the standard model. At the same time, improved modeling for applications in nuclear security, energy, safety, and basic research requires a precision understanding of nuclear cross sections at the few percent level.

We seek therefore to determine a new method to both study physics beyond the standard model and verify and validate light nuclear reaction cross sections. And we aim to do this in such a way that is independent of the cross sections of other reactions, such as fission.

These two apparently disparate problems are therefore intimately related. In order to study beyond-Standard Model physics of the increasingly tightly constrained environment of the early universe, we require highly accurate theoretical models and simulations of its

\footnotetext{
a e-mail: mparis@lanl.gov
}

conditions. The primordial abundances made in the early universe in the process of BBN are becoming an increasingly precise way to do this. The abundances of the light nuclei are currently known at the level of $3 \%-7 \%$ [4] for ${ }^{4} \mathrm{He}$ and $\sim 2 \%$ [2] for deuterium. The observed ${ }^{7} \mathrm{Li}$ abundance [5] in metal-poor halo dwarf stars is currently a factor of 3-4 smaller than that predicted in standard BBN codes; indications of unknown stellar astrophysics [6], however, hint at issues with the extraction of the primordial component from the stellar data. A measurement of interstellar lithium in the small Magellanic cloud [7] indicates consistency with the standard predictions. The nuclear reaction inputs required must be of sufficient precision to satisfy these increasingly rigorous observational constraints.

We first review the standard cosmology and the process of BBN in Sect. 2. We then discuss, in a general way, the $R$-matrix approach to obtain unitary descriptions of scattering and reaction data in a multichannel approach in Sect. 3; specific examples of this approach can be found in the Ref. [8] in this volume. Finally, in Sect. 4, we briefly explain the novel features of our $\mathrm{BBN} /$ neutrino transport 
treatment in the code BURST [9], some of the features of the these simulations, and indicate uses in Verification \& Validation (V\&V) of a proposed new benchmark for light nuclear reaction cross sections.

\section{Review of standard cosmology and BBN}

In the primordial fireball of the early universe, from a time after the big bang of about $\sim 1 \mathrm{~s}$ until the universe is a few minutes old, the light elements, primarily deuterium $D$, helium ${ }^{4} \mathrm{He}\left({ }^{3} \mathrm{He}\right.$, too - but it's not presently observed accurately), and lithium ${ }^{7} \mathrm{Li}$ are formed. During this epoch, the physics of the early universe is that of a homogeneous and isotropic fluid the processes of which manifest the physics of a cooling thermonuclear reactor. Accompanying this physics is the added complexity that arises due to the highenergy photon and neutrino/antineutrino components of the cosmic fluid: the nuclei undergoing fusion reactions and their inverses, both at very high rates - are bathed in high fluxes of these high-entropy particles. The photons are always tightly coupled to both the nuclei and the charged leptons $\left(e^{ \pm}\right)$. Neutrinos transition from a tightly coupled equilibrium regime at comoving temperatures $T_{\mathrm{cm}}$ of 10's of $\mathrm{MeV}$ down to a few $\mathrm{MeV}$ to a decoupled regime through the process of "weak decoupling." Here, the neutrino/antineutrino momentum distributions deviate from Fermi-Dirac equilibrium distributions as they go out of equilibrium with the plasma $\left(\gamma, e^{ \pm}, n, p, \&\right.$ nuclei). Far from being a sharp transition, it occurs over many, $\mathcal{O}(10)$ Hubble times. The epoch of weak decoupling and the BBN epoch, which begins at a temperature of about $1 \mathrm{MeV}$ and continues through $\sim 10 \mathrm{keV}$, are strongly overlapping and tightly coupled.

The nuclear reaction network rate equations evolve the abundances $Y_{\alpha_{1}}$ relative to hydrogen in universal time $t$ as

$$
\frac{d Y_{\alpha_{1}}}{d t} \mathcal{D} \sum_{\alpha_{2}, \beta}\left[-n_{b}\left\langle v_{\beta \alpha} \sigma_{\beta \alpha}\right\rangle Y_{\alpha_{1}} Y_{\alpha_{2}} C n_{b}\left\langle v_{\beta \alpha} \sigma_{\beta \alpha}\right\rangle Y_{\beta_{1}} Y_{\beta_{2}}\right]
$$

where $\alpha, \beta$ are two-body channels of nuclei and other particles $\alpha_{1,2}$, and $\left\langle v_{\beta \alpha} \sigma_{\beta \alpha}\right\rangle$ is the Maxwellian average of the product of the relative velocity with the reaction cross section for the process $\alpha \rightarrow \beta$. Next, we discuss the determination of the $\sigma_{\beta \alpha}$ via the multichannel, unitary $R$-matrix approach. In Sect. 4 we discuss the selfconsistent solution of Eq. (1) in the homogeneous and isotropic Friedmann-Lemaître-Robertson-Walker metric of the early universe self-consistently and concurrently with the collisional Boltzmann equation for the neutrinos/antineutrinos.

\section{3. $R$-matrix description of light nuclei}

The multichannel unitary constraint on the transition $T$ matrix

$$
T_{\beta \alpha}-T_{\beta \alpha}^{\dagger} \mathcal{D} 2 i \sum_{\lambda} T_{\beta \lambda}^{\dagger} T_{\lambda \alpha}
$$

reaction observables are related to the squares of the $T$ matrix elements. Note that while the above relations imply constraints at the level of cross sections (optical theorem; a relation between the total, elastic, and reaction cross sections) they also constrain properties of the $T$-matrix elements at the amplitude level, such as the phases.

The multichannel $R$-matrix approach $[10,11]$ ensures that Eq. (2) are satisfied at the two-body level in parametrizations of scattering and multichannel reaction data. Contrary to alternate approaches (such as approaches that directly parametrize the scattering $S$ matrix or the Heitler or Chew-Mandelstam $K$ matrices) the classic $R$-matrix of Wigner $[12,13]$ considers the solution of the multichannel Schrödinger equation with boundary conditions at finite - as opposed to infinite - distance from the scattering or "interior" region. This results in an expression for the $S$ matrix $(S \mathcal{D} 1 C 2 i T)$ as:

$$
\begin{gathered}
S \mathcal{D} O^{-1} I C 2 i O^{-1} R_{L} O^{-1}, \\
R_{L}^{-1} \mathcal{D} R^{-1} C L-B,
\end{gathered}
$$

where $\rho \mathcal{D} k a$ is a diagonal matrix whose elements are the product of the channel wave numbers $k_{\alpha}$ and the channel radii $a_{\alpha}$, inside which defines the interior, scattering region and $\left.L \mathcal{D} \frac{\rho}{O} \frac{\partial}{\partial \rho} O\right|_{\rho \mathcal{D} k a}$. The $O \mathcal{D} I^{*}$ are the wave functions of two-body channels in the region "exterior" to the scattering region; they are Coulomb functions for charged particle channels and spherical Bessel functions for neutral channels. The real, symmetric matrix $R$ is Wigner's $R$ matrix given as

$$
R_{\beta \alpha} \mathcal{D} \sum_{p} \frac{\gamma_{p \beta} \gamma_{p \alpha}}{E_{p}-E}
$$

it is the Green function of the Schrödinger equation in the presence of the channel surface, $\mathscr{S}$ defined by the union of the locus of points defined by the channel radii. The diagonal matrix $B$ elements are boundary conditions of the states in the interior region with energies $E_{p}$ and reduced widths $\gamma_{p \alpha}$, proportional to the values of the interior region wave functions at the channel surface.

In practice, the $R$ matrix pole positions $E_{p}$ and reduced widths are treated as parameters and varied in a simultaneous minimization of the $\chi^{2}$ of the $R$-matrix model relative to the reaction and scattering observable data. Details of this approach to the phenomenological $R$-matrix approach are given in these proceedings [8].

\section{BBN and neutrino energy transport with BURST}

The self-consistent solution of the BBN reaction network equations [Eq. (1)] and the neutrino energy transport is described in detail in Ref. [9]. The significant advance of the present method is the concurrent and therefore selfconsistent evolution of the neutrino momentum/energy distributions and the nuclear abundances. This results in changes of the primordial abundances from their 'baseline' cases without transport, as shown in Table 1. The results in the table probe the affect that various contributions have to the primordial abundances. The contributions from Coulomb corrections (CC) [14] and zero-temperature radiative corrections (0T) [15] are displayed both singly and in conjunction with each other and the neutrino energy transport corrections, labeled 'Trans.' . This detailed analysis demonstrates a significant enhancement of the 
Table 1. The light element abundances without ('None', baseline) and with neutrino energy transport ('Trans.') and quantum correction ('CC' and 'OT') effects.

\begin{tabular}{lccccccrr}
\hline \hline Processes & $Y_{P}$ & $\delta Y_{P}$ & $10^{5} \times \mathrm{D} / \mathrm{H}$ & $\delta(\mathrm{D} / \mathrm{H})$ & $10^{5} \times{ }^{3} \mathrm{He} / \mathrm{H}$ & $\delta\left({ }^{3} \mathrm{He} / \mathrm{H}\right)$ & $10^{10} \times{ }^{7} \mathrm{Li} / \mathrm{H}$ & $\delta\left({ }^{7} \mathrm{Li} / \mathrm{H}\right)$ \\
\hline None & 0.2438 & 0 & 2.627 & 0 & 1.049 & 0 & 4.277 & 0 \\
CC & 0.2474 & $1.463 \times 10^{-2}$ & 2.647 & $7.898 \times 10^{-3}$ & 1.052 & $2.737 \times 10^{-3}$ & 4.317 & $9.344 \times 10^{-3}$ \\
OT & 0.2442 & $1.454 \times 10^{-3}$ & 2.629 & $7.816 \times 10^{-4}$ & 1.049 & 0.0 & 4.281 & $9.365 \times 10^{-4}$ \\
CC, 0T & 0.2478 & $1.613 \times 10^{-2}$ & 2.650 & $8.719 \times 10^{-3}$ & 1.052 & $3.021 \times 10^{-3}$ & 4.321 & $1.030 \times 10^{-2}$ \\
Trans & 0.2440 & $4.636 \times 10^{-4}$ & 2.636 & $3.686 \times 10^{-3}$ & 1.050 & $1.209 \times 10^{-3}$ & 4.260 & $-3.916 \times 10^{-3}$ \\
CC, 0T, Trans & 0.2479 & $1.644 \times 10^{-2}$ & 2.659 & $1.236 \times 10^{-2}$ & 1.053 & $4.209 \times 10^{-3}$ & 4.304 & $6.231 \times 10^{-3}$ \\
\hline \hline
\end{tabular}

cumulative CC and 0T effects when transport is included. The transport correction (in the fifth row labeled 'Trans.') for the column $\delta Y_{P}$ for ${ }^{4} \mathrm{He}$ is small, as seen in previous work. But the correction for deuterium $\delta(D / H)$ is $\sim 0.4 \%$, which is potentially measurable in forthcoming observations.

\section{References}

[1] K.N. Abazajian et al., ArXiv e-prints (2016), 1610.02743

[2] R.J. Cooke, M. Pettini, R.A. Jorgenson, M.T. Murphy, C.C. Steidel, The Astrophysical Journal 781, 31 (2014)

[3] R.J. Cooke, ApJ 812, L12 (2015)

[4] E. Aver, K.A. Olive, R.L. Porter, E.D. Skillman, J. Cosmology Astropart. Phys. 11, 017 (2013)

[5] M. Asplund, D.L. Lambert, P.E. Nissen, F. Primas, V.V. Smith, The Astrophysical Journal 644, 229 (2006), astro-ph/0510636
[6] J. Meléndez, L. Casagrande, I. Ramírez, M. Asplund, W.J. Schuster, A\&A 515, L3 (2010)

[7] J.C. Howk, N. Lehner, B.D. Fields, G.J. Mathews, Nature 489, 121 (2012), 1207. 3081

[8] G. Hale, M. Paris, Neutron cross sections for carbon and oxygen from new $R$-matrix analyses of the ${ }^{13,14} C$ and ${ }^{17} O$ systems (2016)

[9] E. Grohs, G.M. Fuller, C.T. Kishimoto, M.W. Paris, A. Vlasenko, Phys. Rev. D 93, 083522 (2016), Editors' Suggestion

[10] G. Hale, R.E. Brown, N. Jarmie, Phys. Rev. Lett. 59, 763 (1987)

[11] M. Paris, G. Hale, A. Hayes-Sterbenz, G. Jungman, Nuclear Data Sheets 120, 184 (2014)

[12] E. Wigner, L. Eisenbud, Phys. Rev. 72, 29 (1947)

[13] A.M. Lane, R.G. Thomas, Rev. Mod. Phys. 30, 257 (1958)

[14] C.J. Smith, G.M. Fuller, Phys. Rev. D81, 065027 (2010)

[15] A.F. Heckler, Phys. Rev. D49, 611 (1994) 